\title{
Snap on Smile: Instant Smile
}

\author{
Anubha Sejra ${ }^{1}$, Deepak Raisingani ${ }^{2}$, Ashwini B Prasad ${ }^{3}$, Mohsin Kamaal $^{4}$, Prachi Mital ${ }^{5}$, Rahul Chaudhari ${ }^{6}$
}

\begin{abstract}
No more worry for patient's who were unhappy with the appearance of their smiles. For all those patients who always wanted a new smile but thought that it was beyond their budget, A new option SNAP ON SMILE is answer for all their aesthetic questions.

The snap on smile is a comfortable, removable dental appliance that requires no tooth preparation, no injections and no cements or adhesive. Patient can eat and drink confidently and comfortably while wearing the appliance.

Snap on smile should be targeted to patients who want to quickly feel, look and function better. ${ }^{1}$

Keywords: Cost effective, No tooth preperation, Removal appliance

Journal of Mahatma Gandhi University of Medical Sciences and Technology (2018): 10.5005/jp-journals-10057-0065
\end{abstract}

\section{INTRODUCTION}

Smile plays a predominant role in how a patient perceives himself/ herself. It is an important way of influencing people. Thegesture of smile is involved in expressing several emotions such as happiness, pleasure, humor or greetings, It is very important for the dental practitioner to be thoroughly aware of the intricacies involved in designing a perfect smile. ${ }^{2}$

It is a multipurpose painless removable dental appliance that is comfortable, needs no tooth preparation of existing tooth structure, enhance esthetics and thus simplifies in office process.

Snap on smile appliance is fabricated using a newly improved crystallizedacetyl resin which is more durable, stain-resistant, strong, flexible and functional. It obtains its retention by flexing over the heights and contours of the teeth present in the arch. In case of missing teeth it achieve retention from circumference while in presence of teeth it achieve retention from buccal surface. The appliances neither impinge on the gingiva nor cover the palate. ${ }^{3}$

It resists stains from cigarettes, wine and coffee, and other discoloring foods. Patients can eat and drink confidently and comfortably while wearing the appliance. It last for three to five years and larger if proper care is taken. ${ }^{4}$

\section{Case Description}

A 25-year-old female patient came to the department of conservative dentistry and endodontics with a chief complaint of slight yellow discoloration and malallignment of upper teeth and due to which she was embarrassed by her smile (Fig. 1A).

She went to many dentist and options like bleaching, veneer, crowns and orthodontic treatment was suggested. As patient want noninvasive, immediate, and affordable smile so snap on smile option was suggested to the patient.

The patient underwent a complete examination and records were obtained. It was determined that patient had a mild dental flurosis and angle class $2 \operatorname{div} 3$ malocclusion with deep bite. Patients overall oral hygiene and periodontal health was good with excellent bone support. Veneers and orthodontic treatment was suggested as a permanent treatment option for long term good prognosis.

But above treatment options require more time duration and was expensive and patient was interested in instant gratification

\footnotetext{
$1,4,6$ Resident, ${ }^{2}$ Vice Principal, Professor and Head, ${ }^{3}$ Associate Professor, ${ }^{5}$ Senior Lecturer

${ }^{1-6}$ Department of Conservative Dentistry and Endodontics, Mahatama Gandhi Dental College and Hospital, Jaipur, Rajasthan, India

Corresponding Author: Anubha Sejra, Resident, Department of Conservative Dentistry and Endodontics, Mahatma Gandhi Dental College and Hospital, Jaipur, Rajasthan, India, Phone: 8764252467, e-mail: anubhasejra@gmail.com
}

How to cite this article: Sejra A, Raisingani D, Prasad AB, Kamaal M, Mital P, Chaudhari R. Snap on Smile: Instant Smile. J Mahatma Gandh Univ Med Sci Tech 2018;3(1):36-37.

Source of support: Nil

Conflict of interest: None

where her smile can change within days or weeks rather than in months or years. Patient was very excited to hear an alternative option as a snap on smile.

Both merits and demerits of this treatment approach were explained well in advance to the patient. Next step was collection of intraoral and extraoral photographs for record and analyzing a smile of a patient followed by shape and shade selection of the anterior teeth. There was no tooth preparation or reduction of existing tooth structure.

Another important and next step was impression making, impression of maxillary and mandibular dentitions that clearly duplicate the entire occlusal, buccal and lingual surfaces were taken using addition silicon impression material (Aquasil regular set putty, Dentsply) followed by record of bite registration using bite registration paste (jet bite registration material, ColteneWhaledent, USA). Impression is inspected for bubbles, folds and any other impression errors.

The impressions and records were sent to lab for fabrication of "Snap on smile appliance". Patient was called after few days and snap on smile were snapped over the existing teeth without the need for any adjustment (Fig. 1B). Patient was instructed about handling, maintenance, and about path of insertion of the appliance (Fig. 1C). Patient was very excited as new innovative smile was snapped into place and started smiling without any fear and embarrassment (Fig. 1D).

( ) The Author(s). 2018 Open Access This article is distributed under the terms of the Creative Commons Attribution 4.0 International License (https://creativecommons. org/licenses/by-nc/4.0/), which permits unrestricted use, distribution, and non-commercial reproduction in any medium, provided you give appropriate credit to the original author(s) and the source, provide a link to the Creative Commons license, and indicate if changes were made. The Creative Commons Public Domain Dedication waiver (http://creativecommons.org/publicdomain/zero/1.0/) applies to the data made available in this article, unless otherwise stated. 

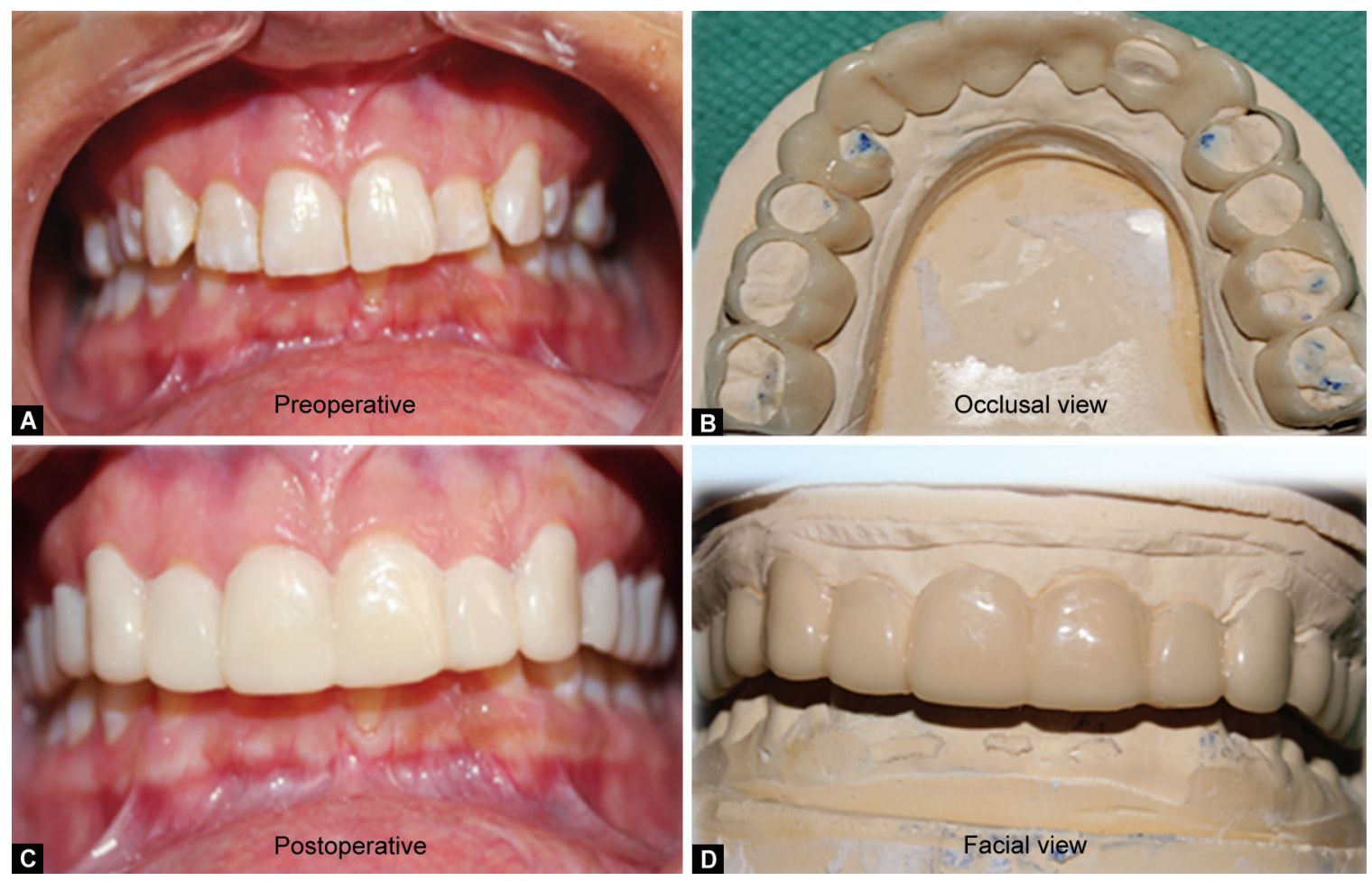

Figs 1 A to D: (A) Preoperative; (B) Occlusal view; (C) Postoperative; (D) Facial view

\section{Discussion}

Snap on smile work on four major modalities which were cosmetic smile enhancement, cosmetic partial denture, implant temporary restoration, and raising vertical dimensions and are mainly indicated in patients who wants a new instant smile for some wedding functions, Class reunion or other important functions on which many people notice your smile. ${ }^{5}$

It can also be used as a transient removable smile until the patient commits to a permanent restorative treatment options like porcelain crowns, veneers. It also act as an economical option within budget when one or missing teeth compromising the patient's smile allowing patients immediate esthetic relief as the patient save funds for the final prosthesis.

It can act as an interim and beautiful smile in between implant placement and implant restoration. It can also be used as passive functional appliance for patients with deep bite and parafunctional habits and is used to raise the vertical dimension. It canalso provide vision to patients whereby patients can see their new smiles and can opt for permanent options.

It is not indicated in patients with severe periodontal diseases, Complete denture patients, patients with high caries index rate or in patients with severe malocclusion teeth and protruding teeth. As the appliance is removable so proper handling and maintenance is very important characteristics for long term usage of the appliance it is advisable not to twist, flex or torque the appliance. Always use both hands while inserting and removing the appliance and establish the correct path of insertion. Cleansing solution should be used to clean the appliance before and after the placement. ${ }^{6}$

Certain limitation of appliance is that the smile is temporary and it won't magically fix missing or broken teeth. Appliance may cause lisping of few words but this problem get resolve as patient wears the appliance regularly.

\section{Conclusion}

Designing a perfect smile comes with considerable concerns as an ideal smile should be composed of components that complement each other and it is crucial for the dental practitioner to be acquainted with the complexities involved in the analysis of smile and the process involved in designing an ideal smile. ${ }^{7}$

The snap on smile offer immediate cosmetic change and is the most compelling aspect of the patients experience, the appliance overcomes many financial and temporal challenges.

\section{References}

1. Introduction to Snap on Smile [Internet]. 2013 Sept. Available from: https://www.nnoha.org/nnoha-content/uploads/2013/09/Snap-onSmile-Todays-Multi-purpose-Restorative-Appliance.pdf

2. Rosenberg J. The Immediate Smile Makeover. Oral Health Journal. 2011:26-32.

3. Téllez LL, Tataje JO, Velásquez JG, Cisneros MH. Rehabilitation with full of metal-ceramic crowns and conventional removable partial prosthesis. OdontologíaSanmarquina. 2009;12(2):78-82.

4. Hill EE, Rubel B, Smith JB. Flexible removable partial dentures: a basic overview. General dentistry. 2014;62(2):32-36.

5. Blackmon LB. Snap-On Smile: a new smile, a happy patient, and further clinical applications. Dentistry today. 2010 Aug;29(8):38.

6. Liechtung M. Snap-on smile: two case studies. Dentistry today. 2006 Apr;25(4):42.

7. Malcmacher L. Snap-on smile: clinical applications. Dentistry today. 2006 Sep;25(9):118-120. 